\title{
Development of PLGA-based itraconazole injectable nanospheres for sustained release
}

This article was published in the following Dove Press journal:

International Journal of Nanomedicine

20 November 2013

Number of times this article has been viewed

\author{
Xiaomei Bian' \\ Su Liang' \\ Jyothy John' \\ Cheng-Hui Hsiao' \\ Xin $\mathrm{Wei}^{2}$ \\ Dong Liang' \\ Huan $\mathrm{Xie}^{1}$ \\ 'Department of Pharmaceutical \\ Sciences, College of Pharmacy and \\ Health Sciences, ${ }^{2}$ Department of \\ Chemistry, College of Science and \\ Technology, Texas Southern University, \\ Houston, TX, USA
}

Purpose: Itraconazole (ITZ) is a synthetic triazole antifungal agent, which is widely used for treatment and prevention of fungal infections. The purpose of this study is to develop ITZ-loaded poly(lactic-co-glycolic acid) (PLGA) nanospheres (PLGA-ITZ-NS) as a new sustained-release formulation for intravenous ITZ administration.

Materials and methods: PLGA-ITZ-NS were prepared by a nanoprecipitation method and optimized by modifying the surfactant poloxamer 188 concentration and PLGA:ITZ ratio. Their physicochemical properties, including size, zeta potential, external morphology and encapsulation efficiency, were characterized by dynamic light scattering (DLS), scanning electron microscopy (SEM) and high performance liquid chromatography (HPLC). The effect of the different selected lyoprotectants with various concentrations on NS particles size and surface charge were also assessed. Rapid and sensitive HPLC and liquid chromatography-tandem mass spectrometry (LC-MS/MS) methods were developed to determine ITZ concentrations in formulation and in rat plasma, respectively. Pharmacokinetics of the optimum PLGA-ITZ-NS formulation was compared with the former commercial Sporanox ${ }^{\circledR}$ injection formulation using rats as the animal model. Noncompartmental pharmacokinetic parameters were obtained by WinNonlin ${ }^{\circledR}$ software.

Results: Optimal PLGA-ITZ-NS had a mean particle size of about $200 \mathrm{~nm}$ with a high homogeneity (polydispersity index $\approx 0.2$ ), favorable zeta potential (approximately -20 to $-30 \mathrm{mV}$ ) and encapsulation efficiency ( $72 \%$ ). In addition, $2 \% \mathrm{w} / \mathrm{v}$ sucrose was selected as a lyoprotectant for NS freeze-drying. The newly developed LC-MS/MS assay was validated and found to be accurate and precise. The in vivo study showed that the NS formulation has a similar systemic bioavailability to Sporanox ${ }^{\circledR}$ while providing a sustained plasma level $(>100 \mathrm{ng} / \mathrm{mL})$ for up to 24 hours after intravenous administration.

Conclusion: Our newly developed PLGA-ITZ-NS has shown great sustained release and comparable bioavailability with Sporanox ${ }^{\circledR}$, therefore having the potential to be an alternative injectable formulation of ITZ.

Keywords: itraconazole, poly(lactic-co-glycolic acid), PLGA, nanoparticle, sustained release, pharmacokinetics, intravenous injection

\section{Introduction}

Itraconazole (ITZ) is a synthetic triazole antifungal agent and is widely used for the treatment and prevention of fungal infections, including blastomycosis, sporotrichosis, and histoplasmosis, especially for systemic infections, such as aspergillosis, candidiasis, and cryptococcosis. ${ }^{1,2}$ Currently, ITZ is also explored as an anticancer agent for patients with metastatic breast cancer and prostate cancer in clinical trials. ${ }^{3}$ Oral ITZ formulation has been broadly applied to treat certain
Department of Pharmaceutical Sciences, College of Pharmacy and Health Sciences, Texas Southern University, 3100 Cleburne Street, Gray Hall II9, Houston, TX 77004, USA

$\mathrm{Tel}+17133134340$

Fax +| $713313109 \mid$

Email xieh@tsu.edu submit your manuscript | www.dovepress.com

Dovepress

http://dx.doi.org// 0.2147/IJN.S54040
International Journal of Nanomedicine 2013:8 452 I-453 I

(c) (i) (5) 2013 Bian et al. This work is published by Dove Medical Press Limited, and licensed under Creative Commons Attribution - Non Commercial (unported, v3.0) License. The full terms of the License are available at http://creativecommons.org/licenses/by-nc/3.0/. Non-commercial uses of the work are permitted without any further permission from Dove Medical Press Limited, provided the work is properly attributed. Permissions beyond the scope of the License are administered by Dove Medical Press Limited. Information on how to request permission may be found at: http://www.dovepress.com/permissions.php 
infections; however, intravenous formulation is necessary for patients who have difficulty swallowing capsules or who need higher ITZ systemic efficacy. The studies of intravenous formulation have been hampered by ITZ's high lipophilicity $(\log \mathrm{P}=6.2)$ and poor aqueous solubility of approximately $1 \mathrm{ng} / \mathrm{mL}$ at a neutral $\mathrm{pH}$ and $4 \mathrm{mg} / \mathrm{mL}$ at $\mathrm{pH} 1 .{ }^{4}$ Sporanox ${ }^{\circledR}$ (Janssen Pharmaceutica NV, Beerse, Belgium) was the only commercial intravenous formulation of ITZ, which was solubilized by $40 \%$ hydroxypropylbeta-cyclodextrin (HP- $\beta-C D)$. However, Sporanox ${ }^{\circledast}$ does have a label warning against its use for renally impaired patients because the clearance of the high concentration of HP- $\beta-C D$ was reduced in those patients, resulting in a higher exposure to HP- $\beta-C D$. Due to this reason, sales and distribution of the Sporanox ${ }^{\circledR}$ intravenous injection in the US were discontinued. In addition, HP- $\beta$-CD has been reported to produce pancreatic adenocarcinoma in a rat carcinogenicity study. ${ }^{5}$ The presence of large amounts of HP- $\beta$-CD limited the use of higher doses and led to the exploration for the alternative parenteral formulations of ITZ. Several intravenous formulations of ITZ, such as the albumin-bound nanoparticles, ${ }^{6}$ lipid nanoparticles,${ }^{5}$ nanosuspension, ${ }^{7,8}$ and nanocrystal, ${ }^{9}$ have been produced without HP- $\beta-C D$; high doses of these formulations (approximately $20-80 \mathrm{mg} / \mathrm{kg}$ ) have been used to obtain a therapeutic efficacy. ${ }^{6,8}$ However, ITZ itself may cause negative inotropic effects. Signs or symptoms of congestive heart failure were mostly reported with a high total daily dose $(400 \mathrm{mg})$ of the Sporanox ${ }^{\circledR}$ injection. ${ }^{10}$ In addition, a sustained release of ITZ has not been completely successful with those new parenteral formulations. Therefore, it is of great interest to develop a new ITZ intravenous formulation without HP- $\beta-C D$ that can provide a sustained release of ITZ and maintain a proper therapeutic level with a lower dose.

Recently, the poly(lactic-co-glycolic acid) (PLGA) nanoparticle-based drug delivery systems have gained a lot of attention due to PLGA's attractive properties. PLGA is one of the most successfully developed biodegradable and biocompatible polymers with the US Food and Drug Administration (FDA) and the European Medicines Agency approval for parenteral administration.

Methods have been well-established to prepare PLGA formulation with many different drugs (hydrophilic or hydrophobic), proteins, peptides, and plasmid DNA. ${ }^{11}$ Moreover, PLGA can protect drugs from quick degradation, modify drug surface properties, and provide targeted delivery to specific organs or cells. ${ }^{12}$ Most importantly, the
PLGA-based nanoparticles have the advantage of a sustained release of the encapsulated therapeutic agents over a period of days or even weeks. ${ }^{11}$ PLGA have been widely applied to encapsulate different drugs, such as ovalbumin, paclitaxel, doxorubicin, flurbiprofen, and others, for the treatment of different pathologies, such as vaccination, cancer, inflammation, cardiovascular disease, etc. ${ }^{12}$ Many of them are in clinical or preclinical trials.

In this paper, we report on ITZ-loaded PLGA nanospheres (PLGA-ITZ-NS) with desired physicochemical properties for a sustained release of ITZ after an intravenous injection. Only a few groups previously reported PLGA-ITZ nanoparticles for in vitro antifungal activity, ${ }^{13-15}$ but their formulations were not systematically characterized for in vivo study, and no pharmacokinetics have ever been completed to explore the formulation's capability of intravenous injection and sustained release. We herein synthesized, characterized, and optimized the formulation for parenteral administration. The resulting optimal PLGA-ITZ-NS were applied for pharmacokinetic study in rats with comparison to Sporanox ${ }^{\circledR}$. A liquid chromatography-tandem mass spectrometry (LC-MS/ MS) assay was developed and validated to analyze the ITZ concentration in rat plasma. The obtained pharmacokinetic parameters indicated that our formulation was bioequivalent to Sporanox ${ }^{\circledR}$, but it provided a successful sustained release of ITZ $>100 \mathrm{ng} / \mathrm{mL}$ for up to 24 hours. This newly developed PLGA-ITZ-NS could be a better alternative to Sporanox ${ }^{\circledR}$ for intravenous injection of ITZ.

\section{Materials and methods Materials}

PLGA (polylactic-glycolic acid, ratio: 50:50, molecular weight 30,000-60,000 Da), poloxamer 188 (Pluronic $^{\circledR}$ F-68), ITZ, Dulbecco's phosphate buffered saline (Life Technologies, Carlsbad, CA, USA), dextrose, sucrose, mannitol, acetone, acetonitrile, and HP- $\beta-C D$ were all purchased from Sigma-Aldrich (St Louis, MO, USA). Sporanox ${ }^{\circledR}$ was purchased from Walgreens (Deerfield, IL, USA). Deionized water (Thermo Fisher Scientific, Waltham, MA, USA) was used throughout the study to prepare the solution and mobile phase.

\section{Preparation of PLGA-ITZ-NS}

The unloaded PLGA nanoparticles and the PLGA-ITZNS were synthesized by a nanoprecipitation method. ${ }^{16}$ In addition, a $1 \%(\mathrm{w} / \mathrm{v})$ PLGA solution was prepared by dissolving the PLGA (50:50) in acetone. To get an optimal concentration of the surfactant used to prepare PLGA 
nanoparticles, $5 \mathrm{~mL}$ of PLGA (1\% w/v) solution was slowly added to four aqueous solutions ( $50 \mathrm{~mL}$ of each) containing $2.5,3,4$, and $10 \mathrm{mg} / \mathrm{mL}$ of nonionic surfactant (poloxamer 188) with stirring. After the optimal surfactant concentration had been determined, various amounts of ITZ were dissolved into three solutions ( $5 \mathrm{~mL}$ of each) of $1 \%(\mathrm{w} / \mathrm{v})$ PLGA in acetone to obtain PLGA:ITZ (w/w) ratios of $5: 1,10: 1$, and 15:1, respectively. The organic phase was added at a constant rate of $0.3 \mathrm{~mL}$ per minute with stirring to $50 \mathrm{~mL}$ of the aqueous phase, containing an optimal concentration of poloxamer 188 . The resulting mixture turned milky instantaneously because of the formation of NS by the solvent displacement and polymer deposition. Acetone was removed by evaporation under vacuum at $60^{\circ} \mathrm{C}$ for 6 hours; about $30 \mathrm{~mL}$ of PLGA-ITZNS suspension was obtained. Finally, the suspension was filtered and centrifuged to remove extra free drug and surfactant.

\section{Characterization of NS}

The particle size, size distribution polydispersity index (PDI), and the zeta potential were measured by dynamic light scattering (DLS) method, using the Zetasizer Nano ZS (Malvern Instruments, Malvern, UK). Each analysis was performed in triplicate.

To obtain the percentage of ITZ encapsulated in PLGA NS, $30 \mathrm{~mL}$ of the PLGA-ITZ-NS suspension in the "Preparation of PLGA-ITZ-NS" section was filtered and centrifuged at 8,000 rpm for 6 minutes. After removing the supernatant, acetonitrile was added to the pellets, followed by sonication for 15 minutes to break the polymer network and release the ITZ. The amount of ITZ was analyzed by the high performance liquid chromatography (HPLC) assay developed by Darouiche et al. ${ }^{17}$ The encapsulation efficiency (EE) was calculated by the following equation:

Encapsulation efficiency \%

$=\frac{\text { Amount of drug in nanosphere }}{\text { Amount of drug used in whole formulation process }} \times 100 \%$

The external morphology of PLGA-ITZ-NS was examined by scanning electron microscopy (SEM). A lyophilized sample was affixed onto an aluminum sample holder with a conductive double-sided carbon tape, followed by imaging with the Hitachi S-4800-II SEM (Hitachi Ltd, Tokyo, Japan) to obtain the SEM images.

\section{Lyophilization}

To discover the best lyoprotectant and optimal concentration for lyophilization, the PLGA-ITZ-NS were freeze-dried in the presence of sucrose, mannitol, dextrose, or HP- $\beta-C D$ at 2,5 , or $10 \% \mathrm{w} / \mathrm{v}$. The control sample was PLGA-ITZ-NS alone, without any lyoprotectant. The samples were lyophilized under 0.018 mbar vacuum (condenser temperature of $-80^{\circ} \mathrm{C}$ ) for 24 hours in a FreeZone Freeze-Dry System (Labconco, Kansas City, MO, USA). The freeze-dried samples were reconstituted in deionized water by shaking. The resuspending time for each sample was recorded. The colloidal NS was then evaluated by the DLS method for particle size, PDI, and zeta potential analysis. The ratio of the final and initial particle size $\left(\mathrm{S}_{\mathrm{f}} / \mathrm{S}_{\mathrm{i}}\right)$ was also calculated.

\section{Analytical methods Assay}

A simple and specific HPLC method developed by Darouiche et al was applied to estimate the ITZ concentration in the NS formulation. ${ }^{17} \mathrm{~A}$ more sensitive and faster LC-MS/MS method was developed to analyze the ITZ in rat plasma. Ketoconazole was used as an internal standard (IS) for this assay. Chromatographic analysis was performed with a 3200 QTRAP LC-MS/MS system (AB SCIEX, Foster City, CA, USA). The mobile phase consisted of $0.2 \%(\mathrm{v} / \mathrm{v})$ ammonia solution and acetonitrile $(20: 80, \mathrm{v} / \mathrm{v})$. The flow rate was $0.5 \mathrm{~mL}$ per minute, using a ZORBAX 300A StableBond-C18 $(4.6 \times 50 \mathrm{~mm}, 3.5 \mu \mathrm{m})$ column (Agilent Technologies, Santa Clara, CA, USA). In addition, $5 \mu \mathrm{L}$ of sample was injected into the column. Each analysis was run for 3.0 minutes. A liquid-liquid extraction and sonication method was used to extract ITZ and IS from the rat plasma. ${ }^{18,19}$ An aliquot of the plasma sample $(50 \mu \mathrm{L})$ was extracted with $200 \mu \mathrm{L}$ of acetonitrile (with $150 \mathrm{ng} / \mathrm{mL}$ of IS) by vortex for 1 minute. After 15 minutes of sonication, the sample was centrifuged at $13,000 \mathrm{rpm}, 4^{\circ} \mathrm{C}$ for 10 minutes. Then, $40 \mu \mathrm{L}$ of aliquot of the supernatant was transferred to an autosampler vial for LC-MS/MS analysis.

\section{Method validation}

The stock solution of ITZ in acetonitrile $(100 \mu \mathrm{g} / \mathrm{mL})$ was further diluted with acetonitrile to make a serial of working standard solutions $(5,10,20,100,500,1,000,1,800$, $2,000 \mathrm{ng} / \mathrm{mL}$ ). The calibration standards were prepared by spiking $5 \mu \mathrm{L}$ of each of the working standard solution into $45 \mu \mathrm{L}$ of blank plasma. The ketoconazole $(150 \mathrm{ng} / \mu \mathrm{L})$ in acetonitrile was applied as IS. The calibration curve was generated by plotting the peak area ratios of ITZ to IS against 
the known concentrations of ITZ. The limit of detection and the limit of quantification were evaluated, based on a signal-to-noise ratio of 6 . The accuracy and precision of the intraday and the interday assay were determined by analyzing samples $(n=6)$ with three different concentrations $(16,150$, and $1,600 \mathrm{ng} / \mathrm{mL}$ ), using a calibration curve constructed on the same day or three sequential days. The acceptable reproducibility concentration was within $\pm 15 \%$. The matrix effect and protein extraction recovery (\%) were performed at concentrations of 10,150 , and $1,600 \mathrm{ng} / \mathrm{mL}$, according to the methods mentioned in our previous study. ${ }^{20}$ The experiment was conducted in triplicates.

\section{Pharmacokinetic study}

Pharmacokinetic studies were performed with adult male Sprague Dawley rats, weighing from 326-355 g. The animal experiment was approved by the Institutional Animal Care and Use Committee at Texas Southern University. The jugular veins of the rats were cannulated under anesthesia, using a cocktail of ketamine-acepromazine-xylazine $(50: 3.3: 3.3 \mathrm{mg} /$ $\mathrm{kg}$ ) the day before the study. Blank blood samples were collected from each rat immediately before dosing. One group of rats $(\mathrm{n}=5)$ received $5 \mathrm{mg} / \mathrm{kg}$ dose of PLGA-ITZNS (approximately $1.7 \mathrm{~mL}$ at $0.985 \mathrm{mg} / \mathrm{mL}$ ) through an intravenous infusion using a 23-gauge needle. Another group of rats $(\mathrm{n}=4)$ was administered with a $10 \mathrm{mg} / \mathrm{kg}$ dose of Sporanox ${ }^{\circledast}$ (approximately $1 \mathrm{~mL}$ at $3.333 \mathrm{mg} / \mathrm{mL}$ ) at the same condition. No syringeability problem was observed for both formulations. Blood samples $(250 \mu \mathrm{L})$ were collected with heparin-treated syringes at 10 minutes, 30 minutes, 1 , $2,4,6,8,12,24,36$, and 48 hours postinjection. The blood samples were centrifuged at 13,000 rpm for 30 seconds, and the supernatants (plasma) were obtained and immediately stored at $-80^{\circ} \mathrm{C}$ until LC-MS/MS analysis.

After analysis, GraphPad Prism5 was applied to obtain curve fit of the plasma concentrations versus time (GraphPad Software, Inc, La Jolla, CA, USA). Statistical interpretations of the data were applied using SYSTAT 11 (Systat Software Inc, Chicago, IL, USA). Sample data were expressed as means \pm standard (SD). Prior to the conduct of any statistical test, the Levene's test was run to check homogeneity of variances in the individual groups. If the variances associated with any two mean values were statistically found to be homogeneous, then the Student's $t$-test was used to determine the statistical significance of pharmacokinetic parameters between the PLGA-ITZ-NS group and the Sporanox $^{\circledR}$ group. The level of significance was set at $P<0.05$. The plasma concentration-time data were pharmacokinetically analyzed separately for each rat via WinNonlin ${ }^{\circledR} 3.0$ (Certara, St Louis, MO, USA) by the noncompartmental method. The parameters thus obtained were the terminal elimination half-life, the total area under the plasma drug concentration-time curve, the mean residence time, the systemic or plasma clearance, and the apparent volume of distribution.

\section{Results \\ NS formulation, optimization, and characterization}

To discover the optimal concentration of the stabilizer used in the NS formulation with the nanoprecipitation method, the PLGA concentration $(1 \% \mathrm{w} / \mathrm{v})$ was kept constant, and the four different poloxamer 188 concentrations were used to prepare unloaded PLGA nanoparticles. As shown in Table 1, the zeta potentials of all the four preparations were between $-22 \mathrm{mV}$ and $-28 \mathrm{mV}$, which can prevent aggregation by repulse particles with each other. When the poloxamer 188 concentration was $3 \mathrm{mg} / \mathrm{mL}$, the optimal PLGA-ITZ-NS formulation was obtained with the smallest particle size $(189 \pm 5 \mathrm{~nm})$ and PDI $(0.08 \pm 0.02)$. Therefore, $3 \mathrm{mg} / \mathrm{mL}$ of poloxamer 188 was chosen for the preparation of PLGAITZ-NS during the whole study.

The properties of PLGA-ITZ-NS synthesized with different PLGA: ITZ (w/w) initial ratios were summarized in Table 2. Again, all the preparations had negative zeta potentials, which can provide steric stability to the particles. PLGA-ITZ-NS, with a ratio of 5:1 and 10:1, gave similar small particle sizes, but the latter had lower PDI and higher EE, which were more preferable. NS, with a ratio of 15:1, provided similar EE as NS with its ratio of 10:1, but the particle size was larger. Therefore, the PLGA-ITZ prepared with an initial ratio of 10:1 was chosen as the optimal formulation when considering particle size, PDI and EE comprehensively, and was utilized for the formulation of all the following studies.

Table I Physicochemical properties of unloaded PLGA nanoparticles stabilized by different concentrations of poloxamer 188

\begin{tabular}{llll}
\hline $\begin{array}{l}\text { Poloxamer } 188 \\
(\mathbf{m g} / \mathbf{m L})\end{array}$ & $\begin{array}{l}\text { Particle size } \\
(\mathbf{n m})\end{array}$ & PDI & $\begin{array}{l}\text { Zeta potential } \\
(\mathbf{m V})\end{array}$ \\
\hline 2.5 & $299 \pm 33$ & $0.45 \pm 0.08$ & $-28 \pm 1$ \\
3 & $189 \pm 5$ & $0.08 \pm 0.02$ & $-22 \pm 0$ \\
4 & $232 \pm 19$ & $0.07 \pm 0.04$ & $-24 \pm 0$ \\
10 & $48 I \pm 41$ & $0.29 \pm 0.03$ & $-23 \pm 1$ \\
\hline
\end{tabular}

Note: $3 \mathrm{mg} / \mathrm{mL}$ was selected as optimal surfactant concentration to prepare PLGAITZ-NS for the remaining studies.

Abbreviations: PLGA, poly(lactic-co-glycolic acid); PDI, polydispersity index; PLGA-ITZ-NS, itraconazole-loaded poly(lactic-co-glycolic acid) nanospheres. 
Table 2 Physicochemical properties of PLGA-ITZ-NS with different PLGA:ITZ initial ratios

\begin{tabular}{lllll}
\hline $\begin{array}{l}\text { PLGA:ITZ } \\
(\mathbf{w} / \mathbf{w})\end{array}$ & $\begin{array}{l}\text { Particle size } \\
(\mathbf{n m})\end{array}$ & PDI & $\begin{array}{l}\text { Zeta potential } \\
(\mathbf{m V})\end{array}$ & EE\% \\
\hline $5: 1$ & $175 \pm 5$ & $0.41 \pm 0.03$ & $-17 \pm 1$ & $61 \pm 4$ \\
$10: 1$ & $178 \pm 6$ & $0.19 \pm 0.03$ & $-20 \pm 1$ & $72 \pm 1$ \\
$15: 1$ & $191 \pm 2$ & $0.14 \pm 0.02$ & $-30 \pm 1$ & $73 \pm 1$ \\
\hline
\end{tabular}

Note: Ratio of 10:I was then selected to prepare PLGA-ITZ-NS for the remaining studies.

Abbreviations: PLGA-ITZ-NS, itraconazole-loaded poly(lactic-co-glycolic acid) nanospheres; PLGA, poly(lactic-co-glycolic acid); ITZ, itraconazole; PDI, polydispersity index; EE, encapsulation efficiency.

\section{Lyophilization and optimization of lyoprotectant}

The control sample of PLGA-ITZ-NS that was freeze-dried without any lyoprotectant was found mostly aggregated; therefore, lyoprotectant is necessary for the lyophilization process. Four different types of lyoprotectants and three different concentrations were evaluated. The resulting particles were characterized in terms of particle size, PDI, zeta potential, resuspending time, and $\mathrm{S}_{\mathrm{f}} / \mathrm{S}_{\mathrm{i}}$ (Table 3). All the particles had negative zeta potentials, ranging from -22 to $-34 \mathrm{mV}$, so they should be stable. When considering the particle size or the $\mathrm{S}_{\mathrm{f}} / \mathrm{S}_{\mathrm{i}}$ ratio, mannitol was not as good as the other three lyoprotectants. Meanwhile, NS freeze-dried with dextrose had a longer resuspending time. It seemed that $2 \%$ sucrose and $2 \%$ HP- $\beta$-CD provided the most stable, uniform, and smallest particles with the shortest resuspending time. Because of the slow clearance of HP- $\beta-C D$ in Sporanox ${ }^{\circledR}$ and the subsequent concern on the severe renal impairment, ${ }^{6} 2 \%$ sucrose instead of HP- $\beta-C D$ was chosen as a lyoprotectant for freeze-drying PLGA-ITZ-NS, which was then used in the pharmacokinetics study.
The morphology of lyophilized PLGA-ITZ-NS was exhibited by SEM (Figure 1A). It was evident that the particles had spherical shape with a smooth surface. It should also be noted that these particles were fairly uniform in size, and the average particle size was consistent with what was obtained by DLS (Figure 1B).

\section{Analytical methods}

The HPLC method was applied to analyze PLGA-ITZ-NS encapsulation efficiency. The LC-MS/MS assay was used for pharmacokinetics study. Detecting transition ions from a precursor ion to a specific product ion for ITZ was (mass to charge ratio $[\mathrm{m} / \mathrm{z}] 705.3-\mathrm{m} / \mathrm{z} 392.3)$ and ketoconazole (IS) was $(\mathrm{m} / \mathrm{z} 531.2-\mathrm{m} / \mathrm{z} 82.2)$. Figure $2 \mathrm{~A}$ showed a representative HPLC chromatogram of ITZ $(59.5 \mu \mathrm{g} / \mathrm{mL})$ and IS $(144 \mu \mathrm{g} / \mathrm{mL})$. Figure 2B showed a representative LC-MS/MS chromatogram of ITZ and IS in the plasma sample collected from a rat at 36 hours postinjection of PLGA-ITZ-NS (5 mg/kg). ITZ and IS peaks were clearly separated in both chromatograms. The retention times of the IS and ITZ in HPLC chromatograms were 3.15 minutes and 6.36 minutes, respectively; in LC-MS/MS, chromatograms were 1.41 minutes and 1.54 minutes, respectively. These results suggested that both assays were simple, fast, and specific.

The calibration curve of the HPLC assay for ITZ in neat solution has good linearity $\left(R^{2}=0.999\right)$ over the range of $0.23-119 \mu \mathrm{g} / \mathrm{mL}$. The calibration curve of the LC-MS/ MS assay for ITZ in rat plasma is linear $\left(R^{2}=0.999\right)$ over the range of $5-2,000 \mathrm{ng} / \mathrm{mL}$. The lower limit of quantitation of ITZ in the HPLC and LC-MS/MS assay was found to be $250 \mathrm{ng} / \mathrm{mL}$ and $5 \mathrm{ng} / \mathrm{mL}$, respectively. These results indicated

Table 3 Physicochemical properties of freeze-dried PLGA-ITZ-NS with various types and concentrations of lyoprotectants

\begin{tabular}{|c|c|c|c|c|c|c|}
\hline Lyoprotectant & $\mathbf{W} / \mathbf{V}$ & Size $(\mathrm{nm})$ & PDI & Zeta potential $(\mathrm{mV})$ & Resuspending time (s) & $\mathbf{S}_{f} / \mathbf{S}$ \\
\hline \multirow[t]{3}{*}{ Sucrose } & $2 \%$ & $210 \pm 6$ & $0.22 \pm 0.01$ & $-27 \pm 0$ & 2 & 1.08 \\
\hline & $5 \%$ & $229 \pm 9$ & $0.12 \pm 0.01$ & $-24 \pm 0$ & 5 & 1.18 \\
\hline & $10 \%$ & $237 \pm 3$ & $0.09 \pm 0.02$ & $-22 \pm 1$ & 10 & 1.22 \\
\hline \multirow[t]{3}{*}{ Mannitol } & $2 \%$ & $320 \pm 33$ & $0.33 \pm 0.03$ & $-33 \pm 1$ & 2 & 1.6 \\
\hline & $5 \%$ & $277 \pm 6$ & $0.4 I \pm 0.05$ & $-33 \pm 1$ & 2 & 1.42 \\
\hline & $10 \%$ & $312 \pm 14$ & $0.49 \pm 0.04$ & $-23 \pm 1$ & 3 & 1.60 \\
\hline \multirow[t]{3}{*}{ Dextrose } & $2 \%$ & $219 \pm 14$ & $0.18 \pm 0.05$ & $-25 \pm 2$ & 5 & 1.12 \\
\hline & $5 \%$ & $234 \pm 6$ & $0.16 \pm 0.02$ & $-27 \pm 2$ & 20 & 1.20 \\
\hline & $10 \%$ & $226 \pm 8$ & $0.14 \pm 0.06$ & $-28 \pm 1$ & 20 & 1.16 \\
\hline \multirow[t]{3}{*}{$\mathrm{HP}-\beta-\mathrm{CD}$} & $2 \%$ & $195 \pm 3$ & $0.09 \pm 0.01$ & $-34 \pm 1$ & 2 & 1.00 \\
\hline & $5 \%$ & $200 \pm 3$ & $0.08 \pm 0.03$ & $-3 \mid \pm 3$ & 5 & 1.03 \\
\hline & $10 \%$ & $204 \pm 2$ & $0.07 \pm 0.01$ & $-30 \pm 4$ & 10 & 1.0 \\
\hline
\end{tabular}

Note: $2 \%$ sucrose was then selected as optimal lyoprotectant and concentration for NS freeze-drying.

Abbreviations: PLGA-ITZ-NS, itraconazole-loaded poly(lactic-co-glycolic acid) nanospheres; PDI, polydispersity index; $S_{f}$ final particle size; $S_{i}$, initial particle size; HP- $\beta$-CD, hydroxypropyl-beta-cyclodextrin; NS, nanospheres. 

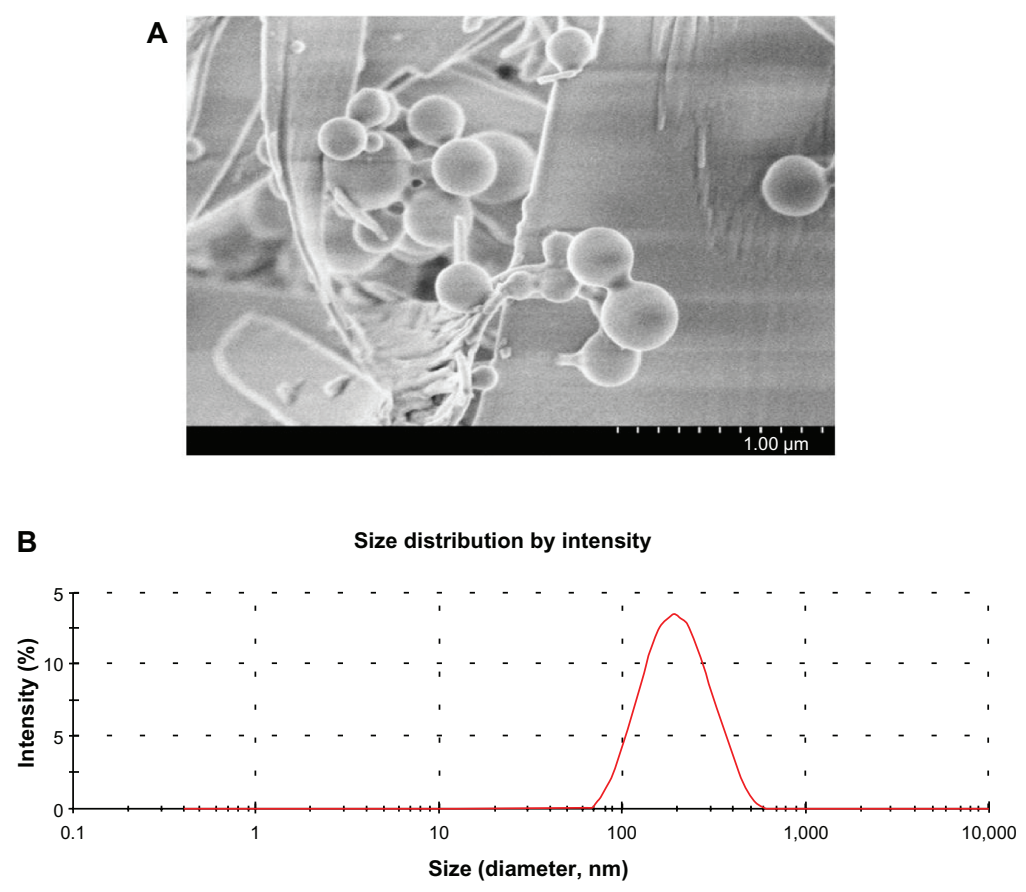

Figure I (A) SEM image and (B) DLS size distribution of PLGA-ITZ-NS.

Abbreviations: SEM, scanning electron microscopy; DLS, dynamic light scattering; PLGA-ITZ-NS, itraconazole-loaded poly(lactic-co-glycolic) acid nanospheres.

that both assays provided good linearity and sensitivity for their specific applications.

Intra- and inter-assay accuracy and precision values for ITZ in rat plasma $(16,150$, and $1,600 \mathrm{ng} / \mathrm{mL})$ were all within $15 \%$, the acceptable limit, suggesting the method was both precise and accurate. The average percentage matrix effects acquired by measuring triplicates of samples at 10,150 , and $1,600 \mathrm{ng} / \mathrm{mL}$ of ITZ in rat plasma were all lower than $15 \%$, and the mean of extraction recovery efficiency was higher than $90 \%$. These results suggested that there were no measurable matrix effects that interfere with ITZ determination in rat plasma via this LC-MS/MS method, and the recovery efficiency rate for ITZ extraction was satisfactory. These method validation results demonstrated that our LC-MS/ MS method for ITZ was optimized for the following pharmacokinetic analysis.

\section{Pharmacokinetic study}

Figure 3 shows the plasma concentration versus time profiles of ITZ after the intravenous administration of our optimal PLGA-ITZ-NS formulation and Sporanox ${ }^{\circledR}$ (control formulation). Sporanox ${ }^{\circledR}$ displayed a biexponential disposition of ITZ; whereas, the PLGA-ITZ-NS formulation showed a rapid initial decline of ITZ followed by sustained plasma ITZ for up to 24 hours before entering its terminal elimination phase. The relevant pharmacokinetic parameters are summarized in Table 4. There are no significant differences on the dose-normalized area under the plasma drug concentration-time curve and clearance between PLGA-ITZ-NS and Sporanox ${ }^{\circledR}$, suggesting a bioequivalence of the two formulations. The difference shown in $\mathrm{V}_{\mathrm{ss}}$ could be due to the special elimination process of the nanoparticles by macrophage phagocytosis. The terminal elimination half-lives were similar between the two formulations, indicating that the plasma samplings were appropriate to catch the terminal elimination phase. It is worth noting that PLGA-ITZ-NS had a significantly longer mean residence time than that of the Sporanox ${ }^{\circledast}$ formulation, which is consistent with the observed sustained plasma ITZ level by PLGA-ITZ-NS formulation.

\section{Discussion}

In the past several years, some new ITZ delivery systems have been developed, such as nanosuspension, ${ }^{13}$ microemulsion, ${ }^{21}$ liposomes, ${ }^{22}$ polymeric micelles, ${ }^{23}$ and nanoparticles,${ }^{5,6}$ to improve ITZ's solubility and bioavailability, thus obtaining better intravenous formulations. Despite those efforts, a sustained release of ITZ has not been completely successful. PLGA, as a biocompatible and biodegradable polymer approved by the FDA, has been widely used for the sustained release of different lipophilic drugs. ${ }^{24}$

Therefore, in this study, we applied PLGA as a drug carrier to obtain a new ITZ intravenous formulation. Several methods are currently employed to formulate PLGA nanoparticles, such as emulsion evaporation, salting out, nanoprecipitation, 


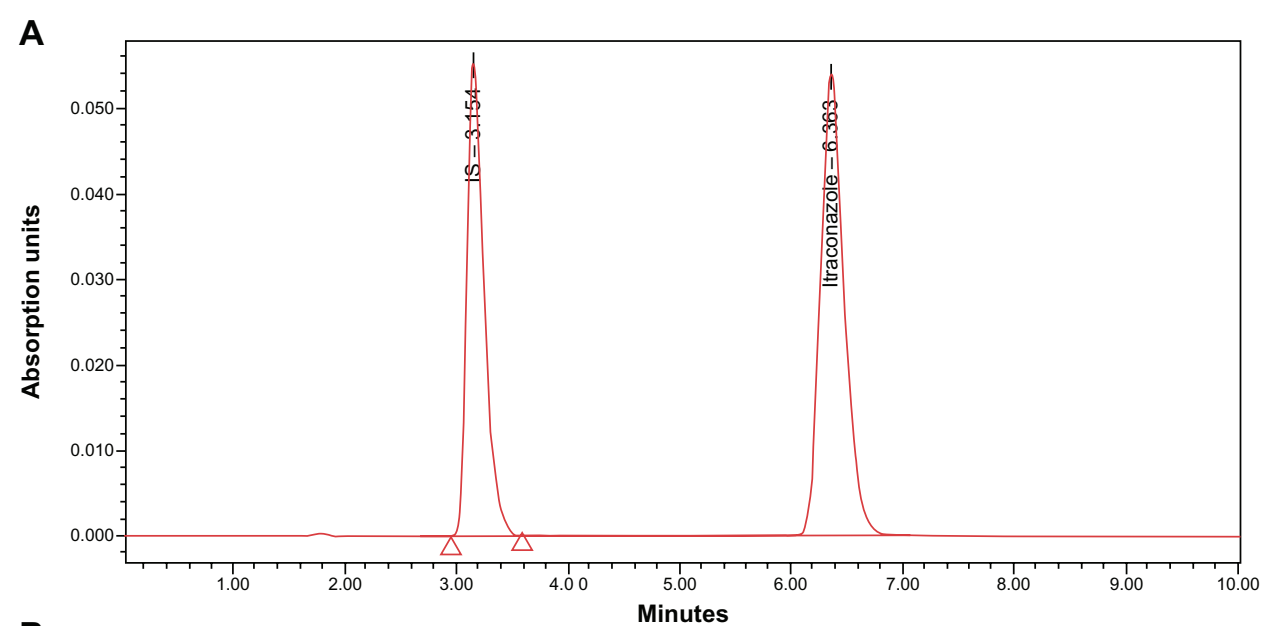

B

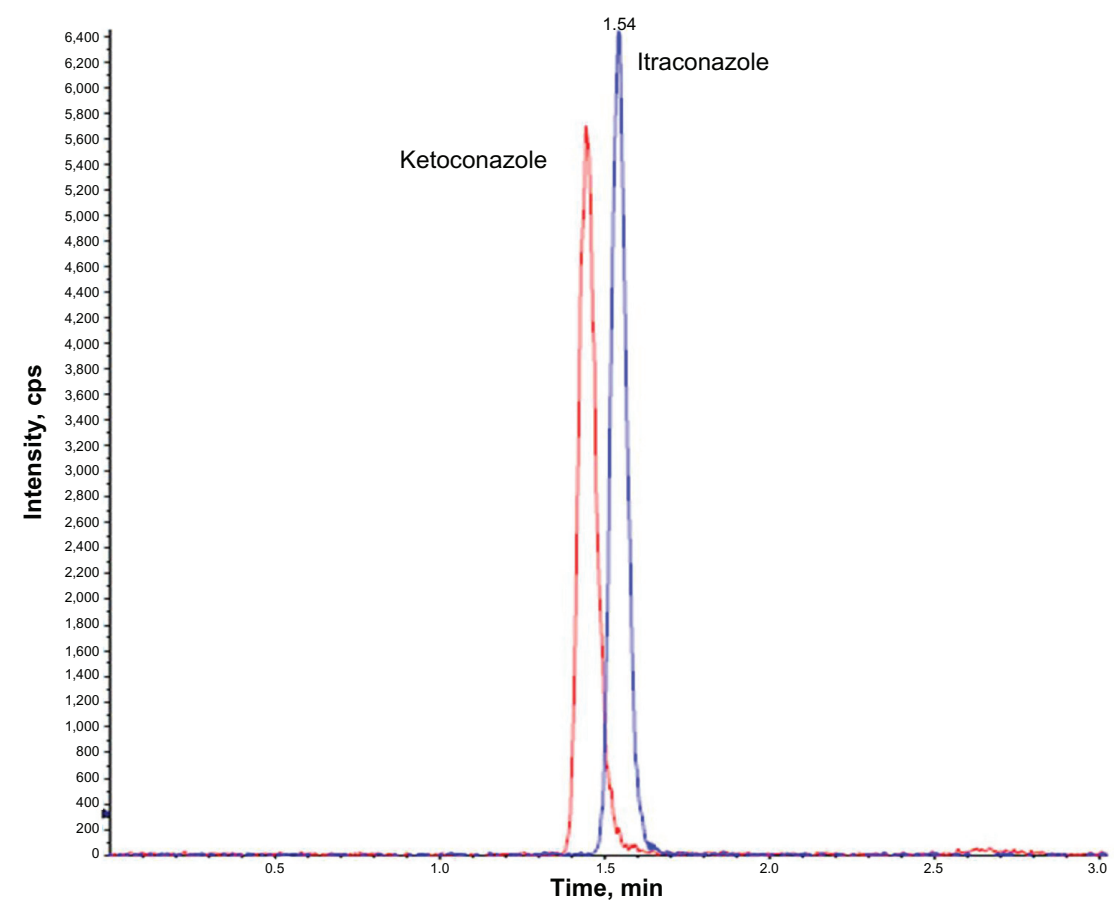

Figure 2 Representative (A) HPLC chromatogram and (B) LC-MS/MS chromatogram of ITZ and ketoconazole (IS).

Abbreviations: HPLC, high performance liquid chromatography; LC-MS/MS, liquid chromatography-tandem mass spectrometry; ITZ, itraconazole; IS, internal standard.

spray drying. ${ }^{25}$ Special attention was focused on using the nanoprecipitation method to encapsulate hydrophobic compounds into the PLGA polymer. This method was first described by Fessi et al and was based on precipitation and a subsequent solidification of the polymer at the interface between a solvent and a nonsolvent. ${ }^{26}$ The nanoprecipitation method can avoid a large amount of toxic solvents and can generate particles with a narrow size distribution without using external energy. ${ }^{25}$ We have adopted this method for the preparation of our PLGA-ITZ-NS.

Physicochemical properties of NPs, such as particle size and distribution, zeta potential, and drug encapsulation rate, are factors that determine the success of a formulation.
The particle size and surface modification play a key role for sustained pharmacokinetics, including the time of absorption, circulation, and distribution, especially for parenteral formulations. Large particles (large than $1 \mu \mathrm{m}$ ) may cause emboli (the diameter of the smallest blood capillaries is $4 \mu \mathrm{m}$ ) and can be taken up by phagocytosis, ${ }^{27}$ while small particles may result in low drug encapsulation and fast drug release. It has been proved that nanoparticles of 100-200 nm in diameter are the most ideal for intravenous application. ${ }^{28}$ As the index of size distribution, the PDI represents the similarity between particles. A large PDI value $(>0.7)$ indicates that the particles have a broad size distribution and are substantially different in size. Samples with a high PDI value may 


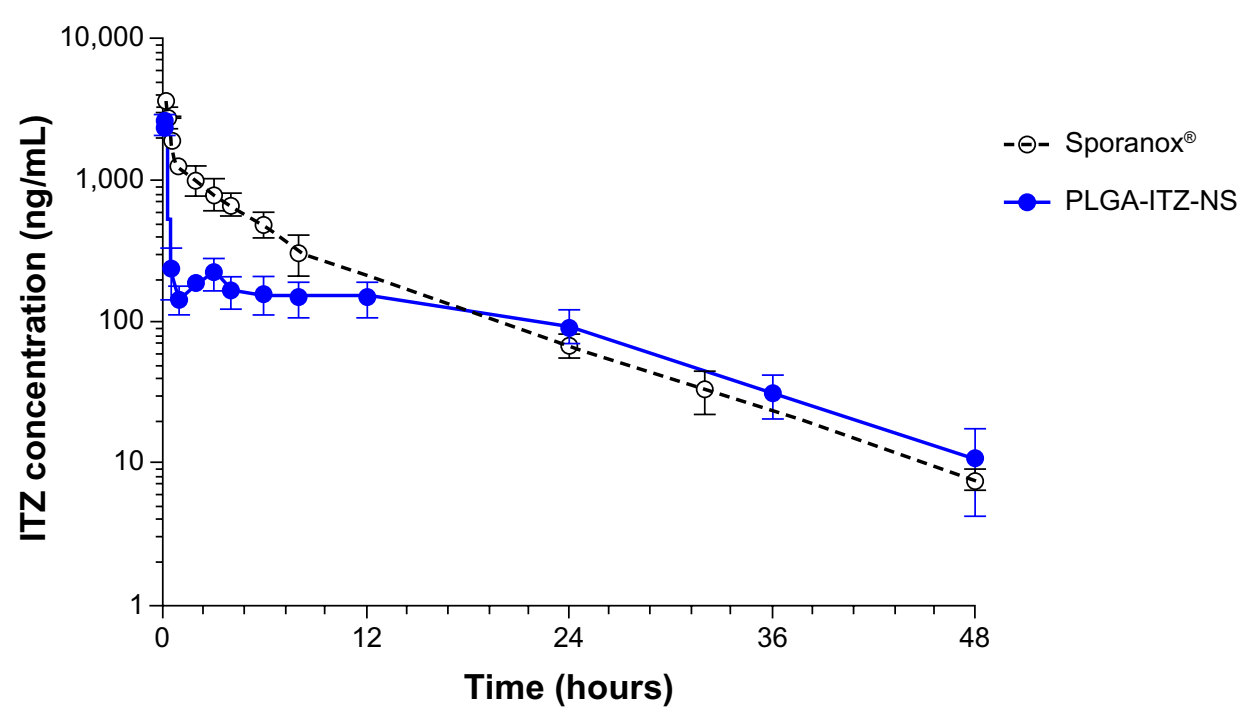

Figure 3 Plasma concentration-time profiles (mean \pm SD) of ITZ after a single intravenous dose of PLGA-ITZ-NS formulation (5 mg/kg) or Sporanox ${ }^{\circledR}$ formulation (control, $10 \mathrm{mg} / \mathrm{kg})$.

Abbreviations: ITZ, itraconazole; PLGA-ITZ-NS, itraconazole-loaded poly(lactic-co-glycolic) acid nanospheres; SD, standard deviation.

contain large particles or aggregates. Unequal particle size can cause pharmacokinetic parameters to be irregular and can affect the therapeutic efficiency of a drug formulation. zeta potential indicates the amount of charges on the surface of nanoparticles. Müller et al demonstrated that a high zeta potential value can ensure a high energy barrier to stabilize nanosuspensions. ${ }^{29}$ Formulation with a high drug encapsulation efficiency can improve its bioavailability, thus making better therapeutic efficacy. All those physicochemical properties were taken into consideration to obtain the optimal ITZ intravenous formulation.

The influence of formulation parameters, such as the type and concentration of stabilizer/surfactant and the PLGA:ITZ ratio on the physicochemical properties of NS, were investigated. A nonionic surfactant presenting at the interface of organic and water phases during nanoparticle

Table 4 Dose-normalized pharmacokinetics parameters of ITZ in plasma after a single intravenous administration of PLGA-ITZ-NS at $5 \mathrm{mg} / \mathrm{mL}$ to rats $(\mathrm{n}=5)$ or Sporanox ${ }^{\circledR}$ at $10 \mathrm{mg} / \mathrm{mL}$ to rats $(\mathrm{n}=4)$

\begin{tabular}{|c|c|c|c|}
\hline Parameter & $\begin{array}{l}\text { PLGA-ITZ-NS } \\
(\text { Dose }=5 \mathrm{mg} / \mathrm{kg})\end{array}$ & $\begin{array}{l}\text { Sporanox }^{\circledR} \\
(\text { Dose }=10 \mathrm{mg} / \mathrm{kg} \text { ) }\end{array}$ & $P$-value \\
\hline $\mathrm{T}_{1 / 2}$ (hour) & $7.7 \pm 1.7$ & $6.9 \pm 1.3$ & 0.768 \\
\hline $\begin{array}{l}\text { AUC } \\
(\mathrm{ng} \times \text { hour } / \mathrm{mL})^{\mathrm{a}}\end{array}$ & $\mathrm{I}, 184.4 \pm 237.4$ & $1,043.3 \pm|8| .7$ & 0.635 \\
\hline MRT (hour) & $12.4 \pm 1.7$ & $7.0 \pm 0.3$ & 0.002 \\
\hline $\mathrm{V}_{\mathrm{ss}}(\mathrm{mL} / \mathrm{kg})$ & $10,610.3 \pm I, 495.7$ & $6,86 I .6 \pm I, 000.3$ & 0.005 \\
\hline $\mathrm{CL}(\mathrm{mL} /$ hour $/ \mathrm{kg})$ & $871.2 \pm 170.0$ & $980.3 \pm 167.7$ & 0.706 \\
\hline
\end{tabular}

Notes: $P$-value was obtained using a Student's $t$-test. ${ }^{2}$ Normalized to I mg/kg dose. Abbreviations: ITZ, itraconazole; PLGA-ITZ-NS, itraconazole-loaded poly(lacticco-glycolic acid) nanospheres; $n$, number of rats used; $T_{1 / 2}$, terminal biological halflife; AUC, total area under the plasma concentration versus time curve; MRT, mean residence time; $\mathrm{V}_{\mathrm{ss}}$, volume of distribution at steady state; $\mathrm{CL}$, clearance. formation is of great importance to reduce the dynamic interfacial tension, thus stabilizing the nanosuspension. Polyvinyl alcohol or Tween ${ }^{\circledR} 80$ are popular stabilizers for the formulation of PLGA nanoparticles; however, it is very difficult to remove the residual stabilizer from the resulting particles' surface, thus causing a biocompatibility issue in the human body. ${ }^{30}$ In our study, poloxamer 188 was selected as stabilizer for PLGA-ITZ formulation because it is a valuable FDA-approved alternative to polyvinyl alcohol with pronounced negative zeta potential. ${ }^{31}$ Stabilizer adsorbed on the nanoparticle surface can contribute to the steric repulsion between particles. As Table 1 shows, PLGA-ITZ-NS stabilized with $3 \mathrm{mg} / \mathrm{mL}$ of poloxamer 188 had a zeta potential of $-22 \mathrm{mV}$, which provided sufficient stability to the formulation. It was also reported that increasing the concentration of the stabilizer can cause resulting nanoparticles to have a larger size. ${ }^{31}$ Our study found the similar trend in particles formed with 3,4 , and $10 \mathrm{mg} / \mathrm{mL}$ of poloxamer 188 , but $2.5 \mathrm{mg} / \mathrm{mL}$ of poloxamer resulted in a larger particle size than that of $3 \mathrm{mg} / \mathrm{mL}$ of poloxamer. This may be because an insufficient amount of stabilizer caused particle aggregation, as also indicated by large PDI.

To obtain an optimal amount of drug encapsulated, different ratios of PLGA-to-drug have been examined. We have evaluated ratios of 5:1, 10:1, and 15:1 w/w, which were similar to the testing ratios of the paclitaxel-loaded PLGA nanoparticles. ${ }^{32}$ The data indicated that increasing the polymer concentration resulted in higher EE, but 10:1 and 15:1 provided almost identical EE (Table 2). Therefore, the PLGA:ITZ ratio of 10:1 was selected. It was close to the 
previously reported ratio of $8: 1 .{ }^{15}$ Our optimal PLGA-ITZNS stabilized with the biocompatible poloxamer 188, had a particle size of $178 \mathrm{~nm}$ (within the optimal approximate 100-200 nm range) with a low PDI (0.19), and had a zeta potential of $-20 \mathrm{mV}$ and high EE $(72 \%)$ - ideal for intravenous administration.

Lyophilization was applied to improve the long-term storage stability of nanosuspensions. However, PLGA nanoparticles have a thin polymeric envelope that may not withstand the stresses of lyophilization - especially, the process of freezing and dehydration. So, lyoprotectants are commonly used to preserve the structure and morphology of colloidal particles and their following reconstitution in aqueous media. We have found that the PLGA-ITZ-NS freeze-dried with $2 \%$ sucrose or $2 \%$ HP- $\beta$-CD had the best physicochemical properties, but because of the concern of using HP- $\beta-C D$ in Sporanox ${ }^{\circledR}$, we decided to use $2 \%$ sucrose for our pharmacokinetic study.

The quantification of ITZ has been achieved by ultraviolet spectrophotometry, ${ }^{15}$ reverse phase high pressure liquid chromatography, ${ }^{6} \mathrm{HPLC} / \mathrm{MS},{ }^{5}$ and LC-MS/MS, ${ }^{33,34}$ among which LC-MS/MS provided highest sensitivity. We have adapted a HPLC assay and developed a LC-MS/MS assay for the measurement of the ITZ amount encapsulated in the PLGA NS and ITZ concentrations in rat plasma, respectively. Both methods have been proved to be fast, accurate, and reliable for their applications. So far, there have only been a few LC-MS/MS methods published for the determination of ITZ. ${ }^{33-35}$ Most of the methods either used a homolog derivative of ITZ as an IS or applied a mobile phase containing acetic acid. ${ }^{33,34}$ To our knowledge, we are the first one to report a LC-MS/MS method for ITZ analysis by using commercially available ketoconazole as IS and a mobile phase containing ammonia. This method was validated in rat plasma with a limit of quantification of $5 \mathrm{ng} / \mathrm{mL}$, acceptable intraday and interday precision and accuracy, as well as matrix effects and extraction recovery.

A comparative pharmacokinetic study between our PLGA-ITZ-NS formulation $(5 \mathrm{mg} / \mathrm{kg})$ and Sporanox ${ }^{\circledR}$ $(10 \mathrm{mg} / \mathrm{kg}$ ) was performed by determining the concentration of ITZ in rat plasma for up to 48 hours after an intravenous administration. Since the sustained release of ITZ from PLGA nanoparticles was expected, we used a lower dose of our formulation. The resulting pharmacokinetic data suggested the two formulations were bioequivalent, but our NS provided a successful sustained release of ITZ. Therefore, it is an improved intravenous formulation than Sporanox ${ }^{\circledR}$. In addition, compared to other drug-loaded PLGA formulations, ${ }^{32}$ our PLGA-ITZ- NS had a similar plasma concentration profile: a rapid initial decline of ITZ in the first couple of hours, followed by a sustained plasma concentration for a much longer period. The rapid decrease of nanoparticles concentration at the beginning is commonly observed and believed to be due to the rapid reticuloendothelial system uptake of nanoparticles after intravenous injection. The sustained ITZ concentration indicated that PLGA played an important role in improving the drug's bioavailability.

In the past five years, some ITZ-loaded nanoparticles have been developed as novel intravenous formulations. For example, Mouton et al reported pharmacokinetics of a nanocrystal formulation of ITZ after single and multiple doses to healthy subjects. ${ }^{9}$ Since it was conducted on humans, the results were not comparable to ours. Rabinow et al evaluated a nanosuspension formulation of ITZ in rats and claimed altered pharmacokinetics compared to Sporanox ${ }^{\circledR 8}$. However, their particles were about $600 \mathrm{~nm}$, which was not in the suitable size range for intravenous administration. Kim et al developed a binary lipid nanoparticles formulation of ITZ for parenteral administration and controlled release. ${ }^{5}$ However, there was no control Sporanox ${ }^{\circledR}$ group in their pharmacokinetic study, nor did they report an ITZ plasma concentration profile. Therefore, it is difficult to compare the data between the two formulations developed by the different labs. Chen et al evaluated a ITZ-loaded human serum albumin nanoparticle formulation in mice. ${ }^{6}$ Although it is difficult to compare directly due to the difference in dose $(20 \mathrm{mg} / \mathrm{kg})$ and specie, ITZ plasma concentration of their formulation was lower than $100 \mathrm{ng} / \mathrm{mL}$ after about 7 hours postinjection, which is much shorter than our sustained release (above $100 \mathrm{ng} / \mathrm{mL}$ for 24 hours). Yi et $\mathrm{a}^{23}$ reported a mixed polymeric micellar formulation of ITZ (particle size in the $20 \mathrm{~nm}$ range), and they performed their pharmacokinetic study in a $5 \mathrm{mg} / \mathrm{kg}$ dose in rats with comparison to Sporanox ${ }^{\circledR}$, so their data were comparable with ours. Their results showed a rapid distribution phase of less than 1 hour, and a terminal elimination half-life of approximately 7-8 hours, which was similar to our NS formulation. However, the pharmacokinetic profiles were very similar between the micellar formulation and Sporanox ${ }^{\circledR}$, which indicates that the micellar formulation did not provide a sustained release of ITZ, and no improvement was achieved. This again illustrates that PLGA can provide a better and a longer sustained release of ITZ than some other nanodrug carriers. Therefore, PLGA-ITZ-NS is a superior sustained-release intravenous formulation. 


\section{Conclusion}

In this study, ITZ was successfully incorporated into PLGA nanoparticles by the nanoprecipitation method. The physicochemical properties were systematically evaluated and optimized to obtain desired PLGA-ITZ-NS around $200 \mathrm{~nm}$ with a narrow size distribution, stable surface charge, and high encapsulation efficiency. Lyophilization was carried out with $2 \%$ sucrose to avoid aggregation. The developed HPLC and LC-MS/MS methods were simple, sensitive, accurate, and suitable for ITZ quantification in a neat solution and rat plasma, respectively. The LC-MS/MS method was further applied to determine ITZ plasma concentration in rats with intravenous injection of our PLGA-ITZ-NS and Sporanox ${ }^{\circledR}$ formulations. The resulting pharmacokinetic parameters were subsequently compared between the two ITZ formulations. Our newly developed PLGA-ITZ-NS formulation without HP- $\beta-C D$ has shown a great sustained release profile with a low-loading dose. Thus, we conclude this nanoformulation appears to be a promising injectable ITZ formulation for future clinical application. Further biodistribution, toxicity, and efficacy studies of this formulation are under investigation in our laboratory.

\section{Acknowledgment}

This project was supported by a grant from National Institute of General Medical Sciences of the US National Institutes of Health (5SC3GM102018).

\section{Disclosure}

The authors report no conflicts of interest in this work.

\section{References}

1. Willems L, van der Geest R, de Beule K. Itraconazole oral solution and intravenous formulations: a review of pharmacokinetics and pharmacodynamics. J Clin Pharm Ther. 2001;26(3):159-169.

2. Odds FC, Oris M, Van Dorsselaer P, Van Gerven F. Activities of an intravenous formulation of itraconazole in experimental disseminated Aspergillus, Candida, and Cryptococcus infections. Antimicrob Agents Chemother. 2000;44(11):3180-3183.

3. ClinicalTrials.gov [webpage on the Internet]. Itraconazole clinical trials. Available from: http://clinicaltrials.gov/ct2/results?term=itraconazole. Accessed October 23, 2013.

4. Peeters J, Neeskens P, Tollenaere JP, Van Remoortere P, Brewster ME. Characterization of the interaction of 2-hydroxypropyl-beta-cyclodextrin with itraconazole at pH 2, 4, and 7. J Pharm Sci. 2002;91(6): 1414-1422.

5. Kim JK, Park JS, Kim CK. Development of a binary lipid nanoparticles formulation of itraconazole for parenteral administration and controlled release. Int J Pharm. 2010;383(1-2):209-215.

6. Chen W, Gu B, Wang H, Pan J, Lu W, Hou H. Development and evaluation of novel itraconazole-loaded intravenous nanoparticles. Int J Pharm. 2008;362(1-2):133-140.

7. McKee J, Rabinow B, Cook C, Gass J. Nanosuspension formulation of itraconazole eliminates the negative inotropic effect of SPORANOX in dogs. J Med Toxicol. 2010;6(3):331-336.
8. Rabinow B, Kipp J, Papadopoulos P, et al. Itraconazole IV nanosuspension enhances efficacy through altered pharmacokinetics in the rat. Int J Pharm. 2007;339(1-2):251-260.

9. Mouton JW, van Peer A, de Beule K, Van Vliet A, Donnelly JP, Soons PA. Pharmacokinetics of itraconazole and hydroxyitraconazole in healthy subjects after single and multiple doses of a novel formulation. Antimicrob Agents Chemother. 2006;50(12):4096-4102.

10. Daily Med, current medication information. Available from: http:// dailymed.nlm.nih.gov/dailymed/lookup.cfm?setid=0453920A-413C4818-8678-029209C5E616. Accessed October 23, 2013.

11. Lü JM, Wang X, Marin-Muller C, et al. Current advances in research and clinical applications of PLGA-based nanotechnology. Expert Rev Mol Diagn. 2009;9(4):325-341.

12. Danhier F, Ansorena E, Silva JM, Coco R, Le Breton A, Préat V. PLGA-based nanoparticles: an overview of biomedical applications. J Control Release. 2012;161(2):505-522.

13. Patel NR, Damann K, Leonardi C, Sabliov CM. Size dependency of PLGA-nanoparticle uptake and antifungal activity against Aspergillus flavus. Nanomedicine (Lond). 2011;6(8):1381-1395.

14. Cunha-Azevedo EP, Silva JR, Martins OP, et al. In vitro antifungal activity and toxicity of itraconazole in DMSA-PLGA nanoparticles. J Nanosci Nanotechnol. 2011;11(3):2308-2314.

15. Patel NR, Damann K, Leonardi C, Sabliov CM. Itraconazole-loaded poly(lactic-co-glycolic) acid nanoparticles for improved antifungal activity. Nanomedicine (Lond). 2010;5(7):1037-1050.

16. Derakhshandeh K, Soheili M, Dadashzadeh S, Saghiri R. Preparation and in vitro characterization of 9-nitrocamptothecin-loaded long circulating nanoparticles for delivery in cancer patients. Int J Nanomedicine. 2010;5:463-471.

17. Darouiche RO, Setoodeh A, Anaissie EJ. Potential use of a simplified method for determination of itraconazole levels in plasma and esophageal tissue by using high-performance liquid chromatography. Antimicrob Agents Chemother. 1995;39(3):757-759.

18. Juhascik MP, Jenkins AJ. Comparison of liquid/liquid and solidphase extraction for alkaline drugs. J Chromatogr Sci. 2009;47(7): 553-557.

19. Tsai YM, Chien CF, Lin LC, Tsai TH. Curcumin and its nano-formulation: the kinetics of tissue distribution and blood-brain barrier penetration. Int J Pharm. 2011;416(1):331-338.

20. Liang S, Bian X, Ma J, et al. Development and validation of a sensitive $\mathrm{LC} / \mathrm{MS} / \mathrm{MS}$ method for the determination of gamma-tocotrienol in rat plasma: application to pharmacokinetic studies. Biomed Chromatogr. 2013;27(1):58-66.

21. Rhee YS, Park CW, Nam TY, Shin YS, Chi SC, Park ES. Formulation of parenteral microemulsion containing itraconazole. Arch Pharm Res. 2007;30(1):114-123.

22. Tang J, Wei H, Liu H, et al. Pharmacokinetics and biodistribution of itraconazole in rats and mice following intravenous administration in a novel liposome formulation. Drug Deliv. 2010;17(4): 223-230.

23. Yi Y, Yoon HJ, Kim BO, et al. A mixed polymeric micellar formulation of itraconazole: Characteristics, toxicity and pharmacokinetics. J Control Release. 2007;117(1):59-67.

24. Huang CL, Steele TWJ, Widjaja E, Boey FYC, Venkatraman SS, Loo JSC. The influence of additives in modulating drug delivery and degradation of PLGA thin films. NPG Asia Materials. 2013;5:e54.

25. Lassalle V, Ferreira ML. PLA nano- and microparticles for drug delivery: an overview of the methods of preparation. Macromol Biosci. 2007;7(6):767-783.

26. Fessi H, Puisieux F, Devissaguet JP, Ammoury N, Benita S. Nanocapsule formation by interfacial polymer deposition following solvent displacement. International Journal of Pharmaceutics. 1989; 55(1):R1-R4.

27. Contado C, Vighi E, Dalpiaz A, Leo E. Influence of secondary preparative parameters and aging effects on PLGA particle size distribution: a sedimentation field flow fractionation investigation. Anal Bioanal Chem. 2013;405(2-3):703-711. 
28. Kulkarni SA, Feng SS. Effects of particle size and surface modification on cellular uptake and biodistribution of polymeric nanoparticles for drug delivery. Pharm Res. 2013;30(10)2512-2522.

29. Müller RH, Jacobs C, Kayser O. Nanosuspensions as particulate drug formulations in therapy. Rationale for development and what we can expect for the future. Adv Drug Del Rev. 2001;47(1):3-19.

30. Sanna V, Roggio AM, Posadino AM, et al. Novel docetaxel-loaded nanoparticles based on poly(lactide-co-caprolactone) and poly(lactideco-glycolide-co-caprolactone) for prostate cancer treatment: formulation, characterization, and cytotoxicity studies. Nanoscale Res Lett. 2011;6(1):260.

31. Vega E, Egea MA, Valls O, Espina M, Garcia ML. Flurbiprofen loaded biodegradable nanoparticles for ophtalmic administration. JPharm Sci. 2006;95(11):2393-2405.

32. Averineni RK, Shavi GV, Gurram AK, et al. PLGA 50:50 nanoparticles of paclitaxel: Development, in vitro anti-tumor activity in BT-549 cells and in vivo evaluation. Bulletin of Materials Science. 2012;35(3): 319-326.
33. Kousoulos C, Tsatsou G, Apostolou C, Dotsikas Y, Loukas YL. Development of a high-throughput method for the determination of itraconazole and its hydroxy metabolite in human plasma, employing automated liquid-liquid extraction based on 96-well format plates and LC/MS/ MS. Anal Bioanal Chem. 2006;384(1):199-207.

34. Manfio JL, dos Santos MB, Favreto WAJ, Weich A, Pugens AM, Donaduzzi CM. Bioequivalence study of two formulations of $100 \mathrm{mg}$ capsule of itraconazole. Arzneimittelforschung. 2010;60(3):157-161.

35. Bharathi DV, Hotha KK, Sagar PV, et al. Development and validation of a highly sensitive and robust LC-MS/MS with electrospray ionization method for simultaneous quantitation of itraconazole and hydroxyitraconazole in human plasma: application to a bioequivalence study. J Chromatogr B Analyt Technol Biomed Life Sci. 2008;868(1-2): $70-76$.
International Journal of Nanomedicine

\section{Publish your work in this journal}

The International Journal of Nanomedicine is an international, peerreviewed journal focusing on the application of nanotechnology in diagnostics, therapeutics, and drug delivery systems throughout the biomedical field. This journal is indexed on PubMed Central, MedLine, CAS, SciSearch $₫$, Current Contents ${ }^{\circledR} /$ Clinical Medicine,

\section{Dovepress}

Journal Citation Reports/Science Edition, EMBase, Scopus and the Elsevier Bibliographic databases. The manuscript management system is completely online and includes a very quick and fair peer-review system, which is all easy to use. Visit http://www.dovepress.com/ testimonials.php to read real quotes from published authors.

Submit your manuscript here: http://www.dovepress.com/international-journal-of-nanomedicine-journal 